\title{
Experimental investigation of vapor bubble growth
}

\author{
J. Pakleza ${ }^{\text {a }}$, M.-C. Duluc ${ }^{\text {a }}$ T. Kowalewski ${ }^{\text {b }}$ \\ a) LIMSI-CNRS, UPR 3251, B.P. 133, 91403 Orsay Cedex, France. \\ b) Polish Academy of Sciences, IPPT PAN, PL 00-049 Warszawa, Poland.
}

\begin{abstract}
An experimental investigation of a vapour bubble growth is performed for water boiling inside a small cube shaped cavity under subatmopsheric pressures. Generation of an isolated bubble is achieved by concentrating heat flux on a small metallic area at the center of the horizontal surface. Bubble growth, recorded using a high speed camera (4500 frames/s), is observed in conditions very close to the ones considered in numerical simulation : axial symmetry, no slip on the heating surface and smooth and regular liquid-vapor interface. Image analysis is performed using a scheme specifically developed for this purpose. The bubble external contour is captured by an edge extracting technique then fitted by Bezier polynomials. The contact line between the wall and the liquid vapor interface is investigated and time evolution of the contact angle and of the bubble base are presented. The velocity at any point of the bubble edge is computed using the optical flow PIV method. Bubble growth mechanisms involved in the present investigation are qualitatively in good agreement with the ones derived from numerical simulation and reported in the literature. This experiment and the associated data analysis represent then a useful means of comparison. Temperature and velocity fields in the liquid surrounding the bubble are determined using liquid crystal tracers and a 3-CCD color video camera. They reveal the adversity to achieve a quiescent and thermally uniform bath during bubble growth experiments.
\end{abstract}

\section{Introduction}

Nucleate boiling is a preferred mode of heat transfer in many industrial techniques due to its high efficiency. Physical mechanism associated with this mode of heat transfer is very complex. Thus, the intensity of heat transfer is usually assessed through theoretical-empirical correlations. Numerous attempts have been performed for more than 50 years in order to predict the growth of an isolated single bubble eluding this way interaction with neighboring bubbles. Bubble growth is derived from two basic mechanisms, evaporation at the bubble base and heat transfer around the bubble. During early stages, bubble expands while a thin liquid film is left on the wall. Heat is transferred from the solid through this microlayer and vaporisation occurs at the liquid-vapour interface creating this way a dry area on the heating surface. The occurrence of the microlayer is enhanced by a hemispherical growth, which is generally encountered during early stages and particularly at low pressures. Simultaneously heat is transferred around the bubble due to the superheating of the surrounding liquid.

Theoretical pioneering models attempted to determine the rate of bubble growth considering a simplified geometry such as a spherical or hemispherical bubble. The complicity of the thermal and hydrodynamic interaction makes analytic solutions of the whole process unattainable. Oversimplified expressions allow nevertheless a fitting description of bubble growth at early or ultimate stages. These are respectively the socalled inertia controlled growth derived by Rayleigh (1917) and the diffusion controlled growth, obtained by Forster and Zuber (1954) or Plesset and Zwick (1954).

Numerical simulations of a single bubble growth have evolved during the last decade. Recent studies carried out by Son et al (1999), Welch (1998) or Fujita and Bai (1998) now make available, for laminar fluid motion, bubble growth from inception to departure without any assumption being made about the bubble shape but axial symmetry. In addition these studies yield velocity and temperature fields in the liquid phase. This is valuable information in order to understand the interaction between the growing bubble and the ambient fluid as suggested by Son et al. (1999). Numerical simulations should also be helpful in order to clarify the role of the solid while solving conjugate heat transfer problems. Time evolution of the so-called triple line, i.e. the contact location between the liquid-vapor interface and the wall, is obviously a major difficulty for numerical investigation. Due to the lack of experimental data, most studies model the triple line using a contact angle of constant value, generally the static contact angle. As well, information is needed when modeling the microlayer region and assumptions have to be made concerning time evolution of the liquid thickness. Experimental data 
are then required in order to validate these models. The bubble shape, the growth rate or the radius of the dried area that are usually employed for comparison are still useful but need to be completed by measurements of additional quantities. Velocity fields may be estimated using tracer particles as in particle image velocimetry (PIV) or particle tracking velocimetry (PTV) techniques. The 3D trajectory and shape of a single air bubble rising in water were measured with these techniques by Hassan et al (2001). Holographic interferometry coupled with high-speed cinematography, used by Chen and Mayinger (1992) in the case of a vapor bubble condensing in a subcooled liquid, allows the determination of temperature fields in the surrounding liquid. Valuable information may also be delivered utilizing thermochromic liquid crystal. Temperature distribution at the back of a thin plate during nucleate boiling was measured using this technique by Kenning and Youyou Yan (1996). The link with the bubble motion was achieved by simultaneous recordings of both events using a high-speed colour camera.

In this paper, an experimental investigation of vapor bubble growth is presented. The experimental conditions are selected so that the observed bubbles are consistent with the means of numerical simulation : bubbles, which may exhibit very various shapes, have an axial symmetry and do not slip on the heating surface. Besides, a smooth and regular liquid-vapor interface is displayed all along the bubble growth. An image analysis is performed and yields an accurate determination of several relevant parameters. Specific attention is devoted to the triple line measuring time evolution of both dried area and contact angle. Velocity and temperature fields in the liquid phase are also visualized seeding the fluid with thermochromic liquid crystals.

\section{Experimental apparatus and procedure}

Isolated bubbles are generated on a horizontal surface inside a $30 \mathrm{~cm}^{3}$ cube shaped cavity. The working fluid is water boiling under subatmospheric pressure. Temperature of the liquid in the internal chamber is controlled with thermostatic fluid flowing through channels drilled inside the cavity walls. The bottom opening is used to mount the heater which consists of a conical brass element electrically heated and embedded in an insulating material (teflon). Stable generation of isolated vapour bubbles is achieved on the brass center of the horizontal surface. The diameter of this boiling area is in the present experiment of $1.42 \mathrm{~mm}$. Temperature is measured in the liquid in the upper part of the cavity and $1 \mathrm{~mm}$ below the boiling surface using K-type thermocouples with an accuracy of $\pm 0.5 \mathrm{~K}$. In the experiments the bulk temperature of the liquid $\mathrm{T}_{1}$ varies in the range $25^{\circ} \mathrm{C}-40^{\circ} \mathrm{C}$. The temperature of the heated surface $\mathrm{T}_{\mathrm{s}}$ is in the range $43^{\circ} \mathrm{C}-72^{\circ} \mathrm{C}$.

The cell construction allows for experimentation in the low-pressure environment. The system pressure was controlled in the range $1 \mathrm{kPa}-10 \mathrm{kPa}$ using a vacuum pump and a $0.5 \mathrm{~m}^{3}$ reservoir. The system used allows for keeping a constant pressure in the cavity for more than 20 hours. In order to perform well-controlled boiling of water in its own vapor environment, special care was undertaken to evacuate the cavity before the experiments. Also the liquid was degassed for about 2 hours before each experimental run.

The five walls of the cube are equipped with optical windows for observation and illumination of the internal chamber. To obtain images of a well-defined bubble interface, back light illumination is applied using the opposite window. A precise description of the bubble growth process is achieved using a Fastcam-Ultima high speed movie camera running at 4500 frames/s. The field of view is approximately $3 \mathrm{~mm} \times 3 \mathrm{~mm}$. Up to 8000 images of $256 \times 266$ pixels are acquired, for each run, by a frame grabber and stored in the computer memory.

The flow visualization around the bubble is performed seeding the fluid with thermochromic liquid crystal tracers. Illumination is realized with a $1 \mathrm{~mm}$ light sheet: a halogen light source is located perpendicularly to the optical axis of the 3 CCD color camera. The 24-bit images of $576 \times 574$ pixels correspond to a field of view of $7.1 \mathrm{~mm} \times 7.1 \mathrm{~mm}$. These images are used for the temperature and velocity evaluation (Particle Image Thermometry and Velocimetry). Particle Image Thermometry is based on the temperature dependent reflectivity of the tracers. When illuminated by white light their color changes from red to blue within a well-defined temperature variation. Unencapsulated tracers with a mean diameter of $25 \mu \mathrm{m}$ and the color play range $35^{\circ} \mathrm{C}$ (red) to $38^{\circ} \mathrm{C}$ (blue) are used in the present experiment. As the volumetric concentration of tracers is very low 
(below $10^{-5}$ ), their effect is supposed to be negligible on the bubble growing process. The local fluid temperature can be determined by relating the color (hue) to a temperature calibration function, obtained from images taken for the same illumination conditions. These tracers are also used to measure the 2-D velocity field distribution by the Particle Image Velocimetry (PIV) method considering two subsequent images. More details about the experimental procedure are available elsewhere (Kowalewski et al. 2000).

\section{Results and discussion}

Experiments were performed with various pressures $(3 \mathrm{kPa}<\mathrm{P}<8 \mathrm{kPa})$, liquid temperatures $\left(25^{\circ} \mathrm{C}<\mathrm{T}_{1}<40^{\circ} \mathrm{C}\right)$ and solid superheats $\left(18^{\circ} \mathrm{C}<\Delta \mathrm{T}_{\mathrm{s}}<32^{\circ} \mathrm{C}\right)$. It was then possible to identify the thermodynamic conditions associated with growth characteristics handled by numerical simulation: smooth and regular contour, axisymmetry, no slip on the wall, no vertical coalescence with the previous bubble. A typical example of such a feature is presented in Fig. 1. It was obtained for a pressure of $4 \mathrm{kPa}$. The liquid temperature $\mathrm{T}_{1}$ was $25.1^{\circ} \mathrm{C}$, which corresponds to a subcooling of $4^{\circ} \mathrm{C}$. The solid superheat indicated by the thermocouple located $1 \mathrm{~mm}$ below the heating surface $\Delta \mathrm{T}_{\mathrm{s}}$ was $21.7^{\circ} \mathrm{C}$.

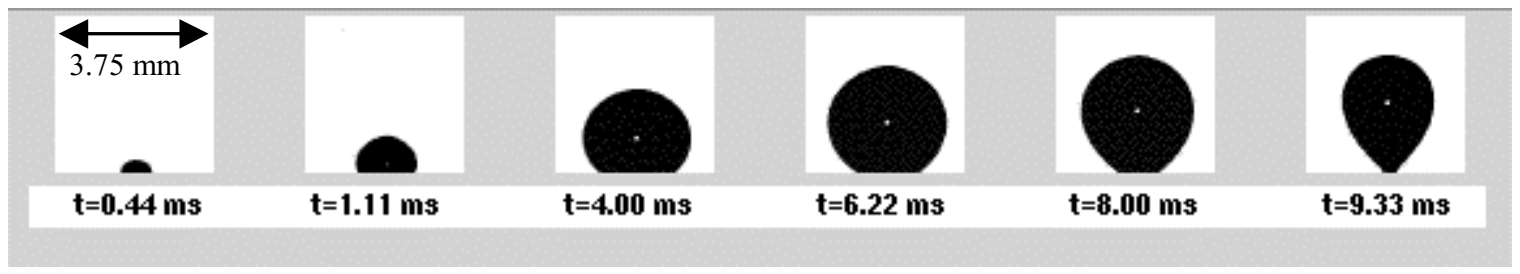

Fig. 1 Growth process of a vapor bubble (water $\mathrm{P}=4 \mathrm{kPa}, \mathrm{T}_{\mathrm{l}}=25.1^{\circ} \mathrm{C}, \Delta \mathrm{T}_{\mathrm{s}}=21.7^{\circ} \mathrm{C}$ )

Due to the back light illumination, cross section of the bubble appears as a dark shadow. The bubble presented in Fig. 1 first displays a hemispherical shape then turns very rapidly $(\approx 0.7 \mathrm{~ms})$ to a truncated sphere. Afterwards, the bubble grows regularly without any significant modification of its form. At time of about $6 \mathrm{~ms}$, the bubble base shrinks and leads finally to the departure process. The whole bubble growth, from time inception to lift-off from the wall takes about $10 \mathrm{~ms}$.
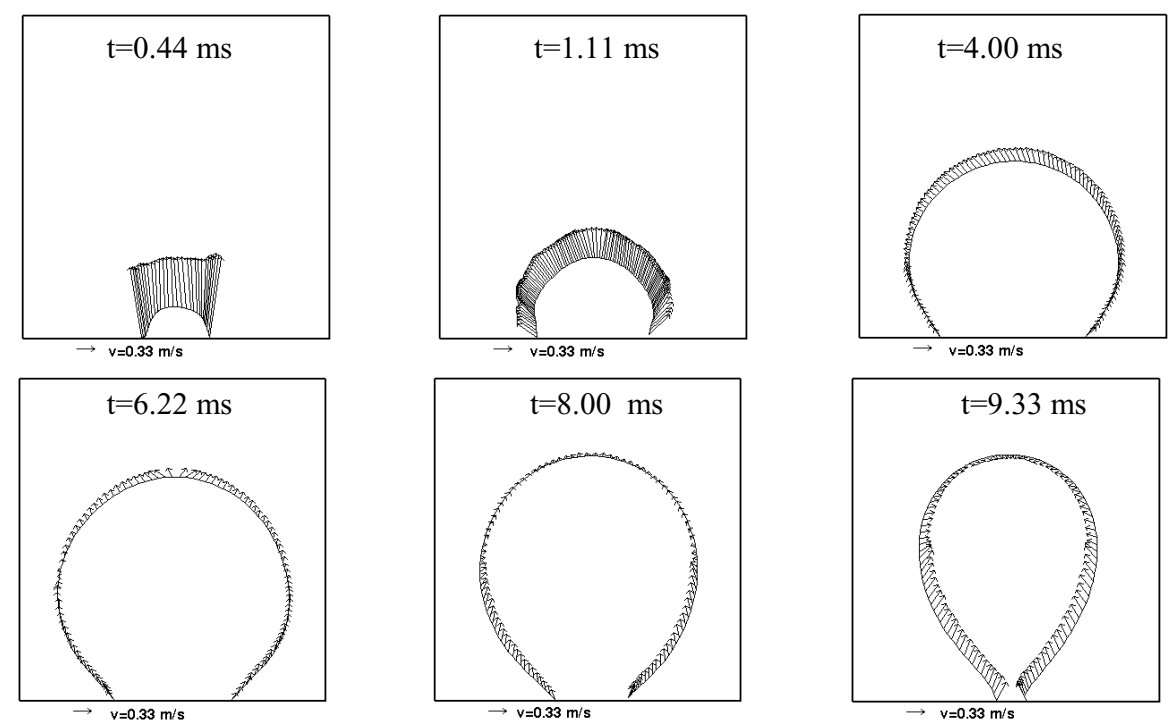

Fig. 2 Velocity at the bubble edge. Computations are performed considering two consecutive frames (time interval $=0.22 \mathrm{~ms}$ )

The optical flow based PIV method (Quénot et al. 1998) is used to evaluate local displacements of the contour from tracer-less images of growing vapor bubbles: for each image, the set of pixels corresponding to the bubble 
contour is first identified. Then, considering two consecutive images separated by a time step of $0.22 \mathrm{~ms}$, the velocity is computed at any point (pixel) of the bubble edge. This calculation is displayed in Fig. 2 for the six bubbles formerly presented in Fig. 1.

First two pictures $(t=0.44 \mathrm{~ms}$ and $\mathrm{t}=1.1 \mathrm{~ms})$ of Fig. 2 show a similar feature, the growth rate of the bubble is nearly uniform. Bubble expands radially outwards displaying this way a hemispherical shape. The highest displacement speed, observed at earlier stages reaches $1.5 \mathrm{~m} / \mathrm{s}$. The third picture displays some noticeable differences : at that time, vaporization is restricted to the upper part of the bubble surface and the speed has significantly decreased (its maximum value, observed at the top, is about $0.33 \mathrm{~m} / \mathrm{s}$ ). The lower part of the edge, corresponding to the bottom third of the bubble height, is no longer exposed to vaporization and looks motionless. This feature progresses with time and the motionless bottom area represents at 6.22 ms more than half the bubble height. The maximum speed has fallen to $0.2 \mathrm{~m} / \mathrm{s}$. The fifth picture ( $\mathrm{t}=8 \mathrm{~ms})$ shows that the lower part of the bubble surface (corresponding to about $70 \%$ of the whole bubble height) is now pushed back by the liquid. Vapor production is confined to the top of the bubble and is associated with a low displacement speed of the contour. The bubble can no longer expand. The last picture $(\mathrm{t}=9.33 \mathrm{~ms})$ shows the bubble just before departure, the contact area being nearly zero. Speed is negative everywhere and its magnitude is not uniform: the bubble cap looks nearly motionless while the bottom edge, where both ends are about to bond together, is submitted to a high speed $(\approx-0.3 \mathrm{~m} / \mathrm{s})$. Therefore, as can be seen in Fig. 3, a flat base is observed after detachment dragging up the liquid from the wall. Numerical simulations derived by Son et al. (1989) or Fujita and Bai (1998) yield results in good qualitative agreement with measurements reported here. They both describe the same feature concerning time evolution of the contact line between the wall and the vapor-liquid interface: it first moves outwards, then inwards as the bubble grows.

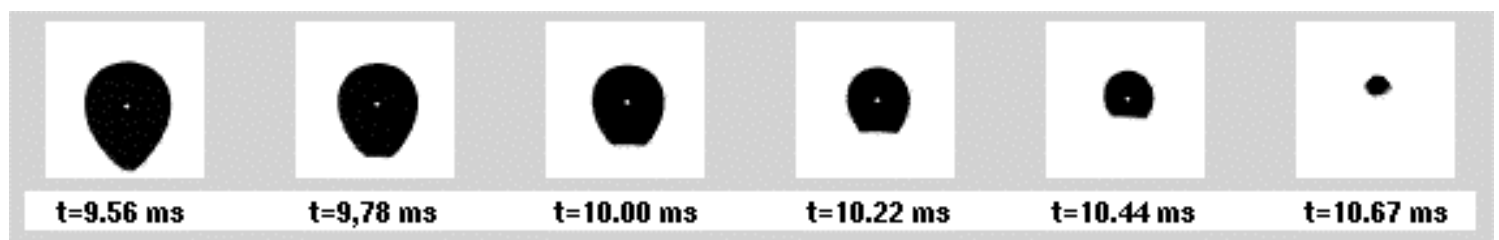

Fig. 3 Time evolution of the vapor bubble after detachment (water $P=4 \mathrm{kPa}, \mathrm{T}_{\mathrm{l}}=25.1^{\circ} \mathrm{C}, \Delta \mathrm{T}_{\mathrm{s}}=21.7^{\circ} \mathrm{C}$ )

An accurate determination of the bubble shape, during the whole growing process, is obtained in the following way: the set of pixels corresponding to the bubble contour is first identified then employed to find a functional interpolation using Bezier polynomials. A smooth representation (up to the second derivative) of the bubble edge is derived and allows further calculation.

The bubble volume is computed assuming an axial symmetry. $D_{e}$, the equivalent diameter of a sphere with the same volume as the growing bubble $V_{b}$, is plotted in Fig. 4. $D_{b}$, the diameter of the bubble base and $H$, its maximum height are also reported on this figure. The growth of the present bubble may be divided in three main stages. A first one where all quantities increase with time. This corresponds to the early period $(0<\mathrm{t} \leq 2.5 \mathrm{~ms})$ where strong vaporization is observed, associated with a hemispherical growth. A second one where $\mathrm{D}_{\mathrm{b}}$ gets smaller while $\mathrm{D}_{\mathrm{e}}$ and $\mathrm{H}$ still increase $(2.5 \mathrm{~ms} \leq \mathrm{t} \leq 7.2 \mathrm{~ms})$. The bubble base starts to shrink but the amount of vapor produced at the bubble cap exceeds the loss at the bottom. This is no longer true during the last period $(7.2 \mathrm{~ms} \leq \mathrm{t} \leq 9.33 \mathrm{~ms}$ ), vapor production is not strong enough to balance the deficit at the base and the bubble diameter $\mathrm{D}_{\mathrm{e}}$ decreases while $\mathrm{H}$ remains nearly constant. The bubble closes down, then lifts off $(9.33 \mathrm{~ms}<\mathrm{t}<9.56 \mathrm{~ms})$. Time histories of both bubble diameter and base diameter look very similar to those obtained by Fujita and Bai (1998) from numerical simulation of a water bubble growth under atmospheric pressure. The maximum value observed for the diameter of the bubble base is in the present case of about $1.5 \mathrm{~mm}$. The metallic hot spot from which bubbles are generated has a diameter of $1.42 \mathrm{~mm}$.

The ratio of $D_{b}$ to $D_{e}$, is plotted in Fig. 5. A regular decrease in time is visible except for both ends. As mentioned above, a very high expansion rate of the bubble base is observed during early stages. Figure 2 (time $=0.44 \mathrm{~ms}$ ) shows that the displacement speed is slightly higher near the wall. As a consequence, a ratio 
$\mathrm{D}_{\mathrm{b}} / \mathrm{D}_{\mathrm{e}}$ greater than unity is derived at that time. The deviation observed at the other end of the chart is due to the high speed involved during the closing process and discussed above.

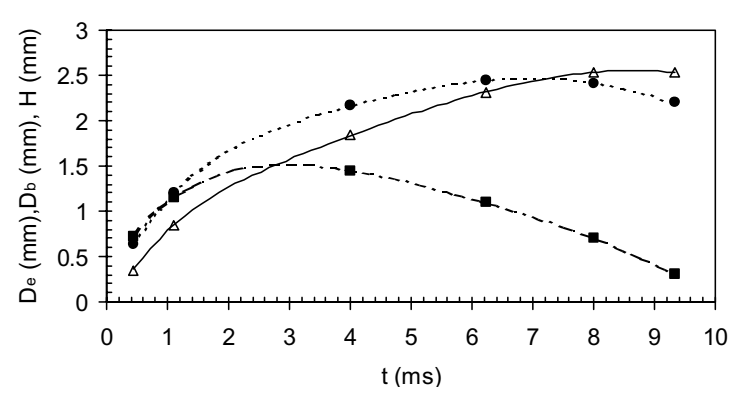

Fig. 4 Times histories of bubble diameter $D_{e}(1)$, base diameter $D_{b}(n)$ and height $H(\Delta)$

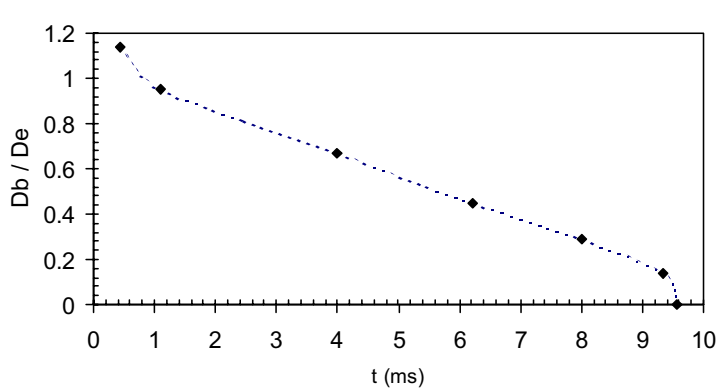

Fig. 5 Time evolution of the ratio $D_{b} / D_{e}$

Left and right contact angles are presented in Fig. 6 . They were calculated considering the apparent angle between the surface and the bubble edge near the wall. Images presented in this paper have a spatial resolution of about $15 \mu \mathrm{m}$ per pixel. Such a phenomenon like microlayer evaporation associated to hemispherical growth is therefore impossible to observe due to the small thickness of this thin liquid film (a few microns as measured by Koffman and Plesset (1983)). Fig. 6 shows that the most significant variations of the contact angle are observed at early and ultimate stages. This is due to the hemispherical growth observed at the very beginning of the bubble growth and to the formation, beyond $8 \mathrm{~ms}$, of a small neck-like structure, less than $300 \mu \mathrm{m}$ in eight.

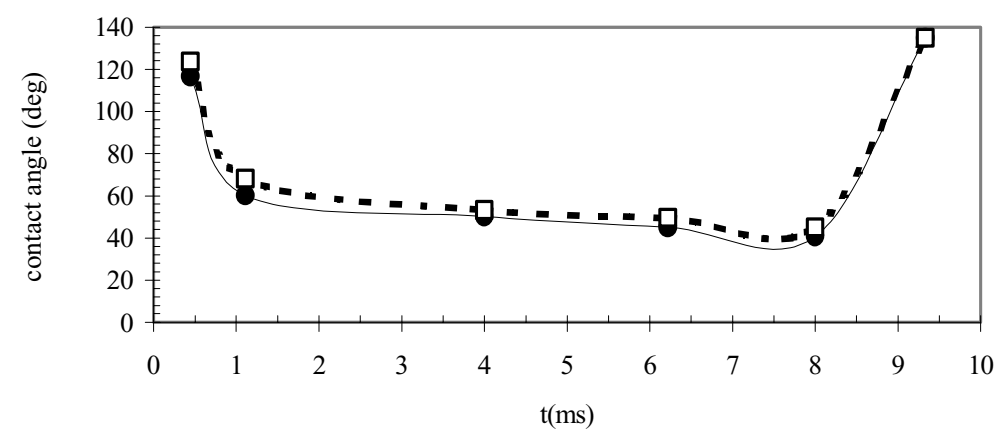

Fig. 6 Time evolution of left (•) and right ( $\square)$ contact angles

The velocity and temperature fields in the liquid phase were investigated with PIV and PIT measurements for slightly different experimental conditions. Recordings are performed using a 3 CCD video camera running at 25 frames/s. The velocity fields are therefore computed considering the motion of liquid crystal particles over a time period of $20 \mathrm{~ms}$. The result of this calculation is presented in Fig. 7. The main feature revealed by these tracers is the presence of some cold liquid flowing down. Its velocity, equal to a few $\mathrm{mm} / \mathrm{s}$, is weaker than the one induced by the bubble growth. The liquid stream is therefore deflected laterally. The examination of the whole video sequence shows that this flow is connected with the presence of convection cells inside the cavity. Despite the use of a regulation device in order to ensure a uniform temperature and motionless bath, velocity and temperature fields are present in the cavity as revealed by these tracers.

\section{Conclusion}

An experimental investigation of vapor bubble growth is carried out. Its aim is to provide data in order to validate numerical models. The image processing developed specifically for this study yields a quantitative 
description of the bubble dynamics at any point of the bubble edge, no assumption being made except axial symmetry. Due to the experimental conditions, bubble growth reported in the present paper, is mainly controlled by two classical mechanisms competing with each other: strong vaporization dominates everywhere on the interface at early stages then condensation occurs at the bubble base that shrinks until bubble lifts-off. Time evolution of the contact angle shows a strong variation of this parameter at the beginning and at the end of the bubble growth. In addition, this investigation shows that the closing down process, leading to bubble departure, induces high velocities at the bubble base. Measurements of the velocity and temperature fields in the liquid using thermochromic liquid crystals and a standard color video camera reveal the occurrence of adverse effects and the difficulty to control experimental surroundings. Additional valuable information should be provided by measuring velocity distribution in the surrounding liquid using the PIV method associated to a high speed camera.

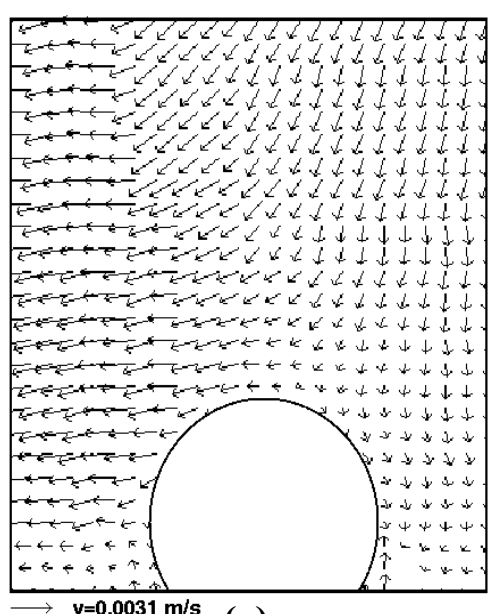

(a)

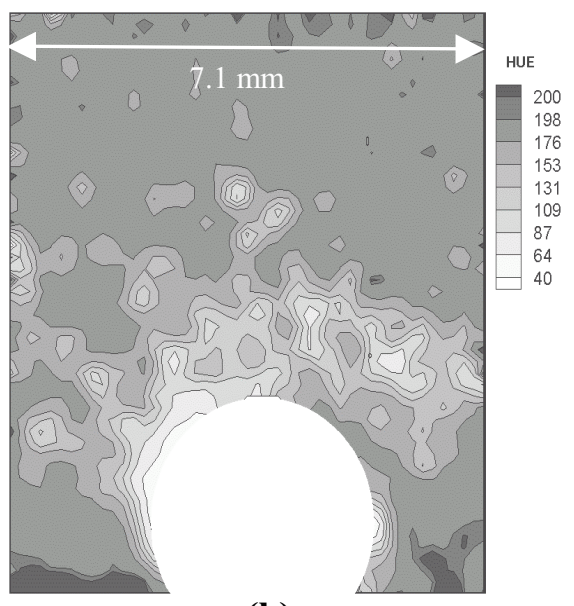

(b)

\section{Fig. 7 Evaluated velocity (a) and temperature (b) fields surrounding the steady vapor bubble visualized with liquid crystal tracers (water $P=6 \mathrm{kPa}, \mathrm{T}_{1}=35.5^{\circ} \mathrm{C}, \Delta \mathrm{T}_{\mathrm{s}}=31.1^{\circ} \mathrm{C}$ ) Temperature calibration yields Hue $=40\left(T_{1}=35.2^{\circ} \mathrm{C}\right)$ and $\mathrm{Hue}=200\left(\mathrm{~T}_{1}=38.1^{\circ} \mathrm{C}\right)$}

\section{References}

Y.M. Chen and F. Mayinger, Measurement of heat transfer at the phase interface of condensing bubbles, Int. J. Multiphase Flow, vol. 18, No. 6, pp. 877-890, 1992.

K. Forster and N. Zuber, Growth of vapor bubbles in superheated liquid, J. Appl. Phys., vol. 25, pp. 474-478, 1954.

Y. Fujita and Q. Bai, Numerical simulation of the growth for an isolated bubble in nucleate boiling, Proc $11^{\text {th }}$ Int. Heat Transf. Conf., vol. 2, pp. 437-442, 1998.

Y.A.Hassan, J. Ortiz-Villafuerte and W. D. Schmidl, Three-dimensional measurements of single bubble dynamics in a small diameter pipe using stereoscopic particle image velocimetry, Int. J. Multiphase Flow, vol. 27, pp. 817-842, 2001.

D.B. R. Kenning and Youyou Yan, Pool boiling heat transfer on a thin plate: features revealed by liquid crystal thermography, Int. J. Heat Mass Tran., vol. 39, No. 15, pp. 3117-3137, 1996.

L.D. Koffman and M.S. Plesset, Experimental observations of the microlayer in vapor bubble growth on a heated solid, J. Heat Transf., vol. 105, pp. 625-632, 1983.

T.A. Kowalewski, J. Pakleza, J.-B. Chalfen, M.-C. Duluc, A. Cybulski, Visualization of vapor bubble growth, $9^{\text {th }}$ International symposium on flow visualization, pp. 176/1-176/9, 2000.

M.S. Plesset and S.A. Zwick, The growth of vapor bubbles in a superheated liquid, J. Appl. Phys., vol. 25, pp. 493-500, 1954.

G. Quénot, J. Pakleza and T.A. Kowalewski, Particle image velocimetry with optical flow, Exp. in Fluids 25, pp. 177-189, 1998.

J.W.S. Rayleigh, On the pressure developed in a liquid during the collapse of a spherical cavity, Phil. Mag., vol. 34, pp. 94-98, 1917.

G. Son, V.K. Dhir and N. Ramanujapu, Dynamics and heat transfer associated with a single bubble during nucleate boiling on a horizontal surface, J. Heat Transf., vol. 121, pp. 623-631, 1999.

S. W. J. Welch, Direct simulation of vapor bubble growth, Int. J. Heat Mass Tran., vol. 41, pp. 1655-1666, 1998. 
